\section{THE NEW ROYAL VICTORIA HOSPITAL, BELFAST.}

BY J. W. BYERs, M.A., M.D. R.U.I,

PROFESSOR OF MIDWIFERY IN QUEEN'S COLLEGE, BELFAST; AND PHYSICIAN FOR DISEASES OF WOMEN TO THE ROYAL VICTORIA HOSPITAL.

Is the planning, construction, and furnishing of a large hospital associated with a medical school in a city like Belfast, the main considerations to be kept in view are as follows:-

1. The institution should be sufficiently large to be self contained, it should be accessible, and it should occupy a position in which it cannot be deprived of light and air by contiguous buildings, or be fouled by the smoke from adjacent manufactories. which the principal considerations are perfection of work and its accomplishment with care and despatch, while at the same time this maximum of efficiency should be secured at the minimum of cost. How far has the new Royal Victoria Hospital, erected as the outcome of a movement which began in December, 1896, during the mayoralty of the Right Hon. W. J. Pirrie to do honour to the Diamond Jubilee of Her late Majesty Queen Victoria, fulfilled the canons laid down?

1. Thanks to the generosity of the corporation of the city of Belfast the site of the new hospital at Grosvenor-street comprises six acres of land at present, while an additional six acres will be available when the patients at the old asylum are removed. This is an unusually large area for an institution within a city to find at its disposal. The district also is an elevated one with good mountain air. At present the site is bounded on two sides by streets, though it enjoys

FIG. 1.

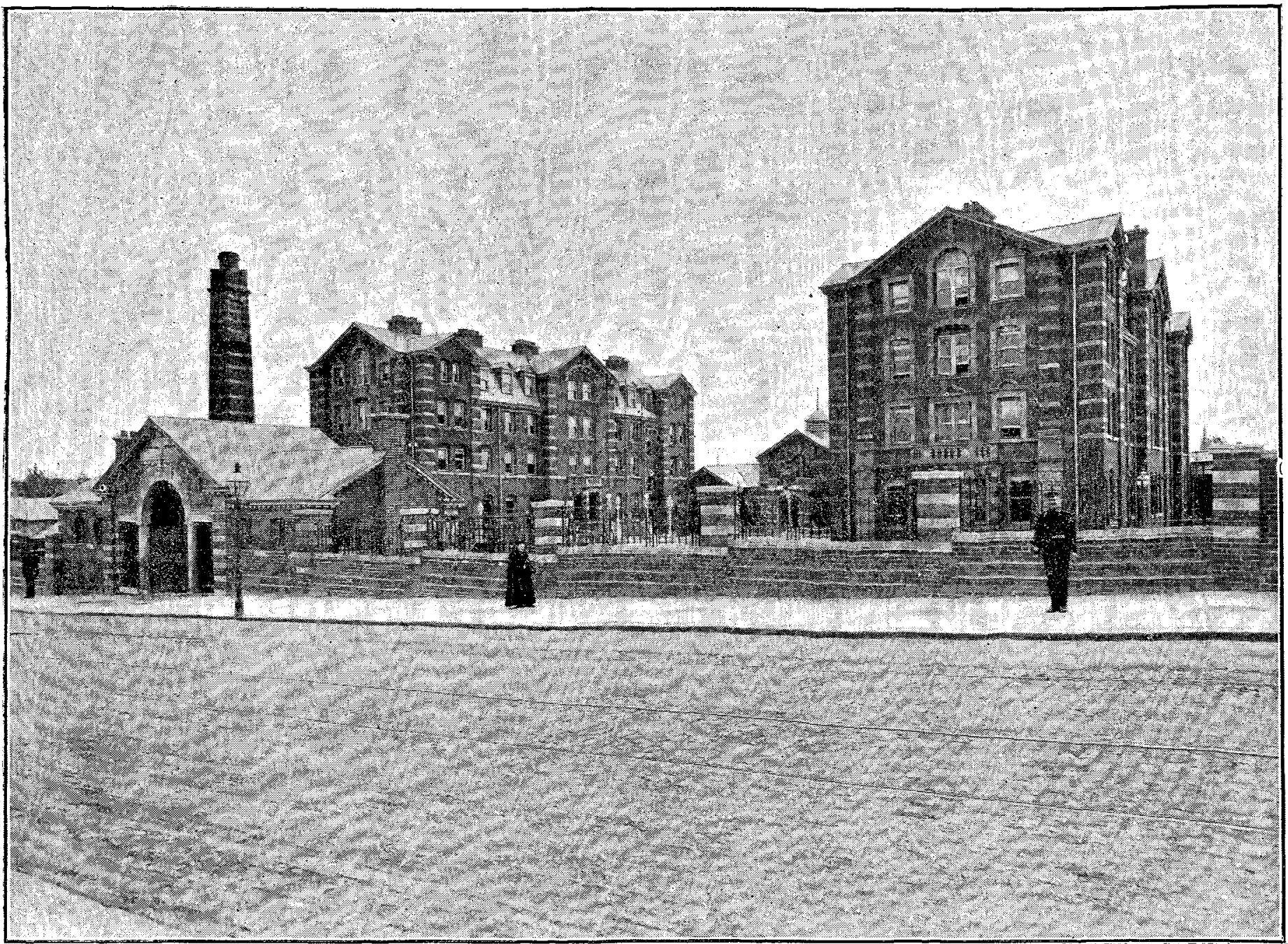

Main entrance of the New Royal Victoria Hospital, flanked by the two administrative blocks.

2. It should have as its object to secure the maximum of comfort for the patients and it should be so constructed that they are placed as regards sanitation, heating, and ventila. tion in a pure or aseptic environment.

3. It should be easy of administration in regard to dietetic and nursing arrangements.

4. It should be so planned that the medical and surgical staff may be able to employ all the most recent scientific advances in every department of medicine, however special, that have been designed for the preservation of life and for the alleviation of suffering, while at the same time affording facilities for the practical teaching of those who will be the practitioners of the future.

5. The arranging of the buildings should, if possible, be finally referred to one or two experts who have a practical knowledge of construction and of modern hospital administration.

In a word, the ideal hospital should be planned and designed on the lines of a well-arranged manufactory in the special advantage of having at its northern aspect no buildings but only the beautitul Dunville Park. On the south side, where the wards are located, there is plenty of space for recreation grounds. The situation is a convenient one on a main road-artery of the city with a tram-line.

2. As regards internal construction, plumbing, heating, and ventilating the hospital is as near perfection as care and expert knowledge can make it. It is heated and ventilated on the Plenum system, which has been adopted in its most complete form and which will provide an equable temperature in summer or winter and will protect the patients from being affected by those sudden and protean changes so common in our variable climate, while at the same time by the constant change of pure air, cold in summer and heated in winter, the patients will live in a comparatively aseptic atmosphere.

The ventilation is managed as follows. At the east termination of the main corridor is placed the air-intake and under this corridor the air-duct runs its whole length and 
beyond it to supply two small isolation buildings. At its hospitals, a plan has been matured which promises success. entrance the intake is 20 feet high by nine feet in breadth, while at its termination it is nine feet in breadth by a few feet high. The duct divides into the shape of an inverted $\lambda$ towards the east, each arm of the $\lambda$ ending in a circular aperture slightly larger than the rotating fan fixed in each apsrture. These great fans are ten feet four inches in diameter, with six blades, and each is capable of driving $10,000,000$ cubic feet of air per hour. The machinery lies in the space between the arms of the $\lambda$ and both engines and fans are duplicated. One propellor is used at a time, but the other can be put into use in a few seconds. Round each fan a large room is built with openings, and arranged in rows vertically in it are a large number of steam pipes. The air washed by passing through wet curtains of coir matting and heated by the pipes is, when thus warmed and purified, driven up the air-duct from which branch ducts run under the various corridors. Mrs. Pirrie is responsible for it. The patients' food will be carved in a second room off the main kitchen, it will then be placed in closed tins with hot-water jackets, put into trucks, raised to the main corridor by small food-lifts, and never opened until it is by the patient's side. In this way expedition in transit is combined with warmth of the food. The ward kitchens are furnished with racks like those used in a ship, so that every cup, plate, and utensil can be replaced in order and the matron in taking stock can see at a glance if any article is missing.

4. From the medical and surgical standpoint the arrangements of the hospital are admirable, representing the last word in hospital construction. Each of the four physicians and four surgeons will have a set of two wards, male and female, side by side. Running off each of these wards are two small wards and a kitchen. Each surgeon has his own operating theatre, the corresponding room assigned to the

FrG. 2.

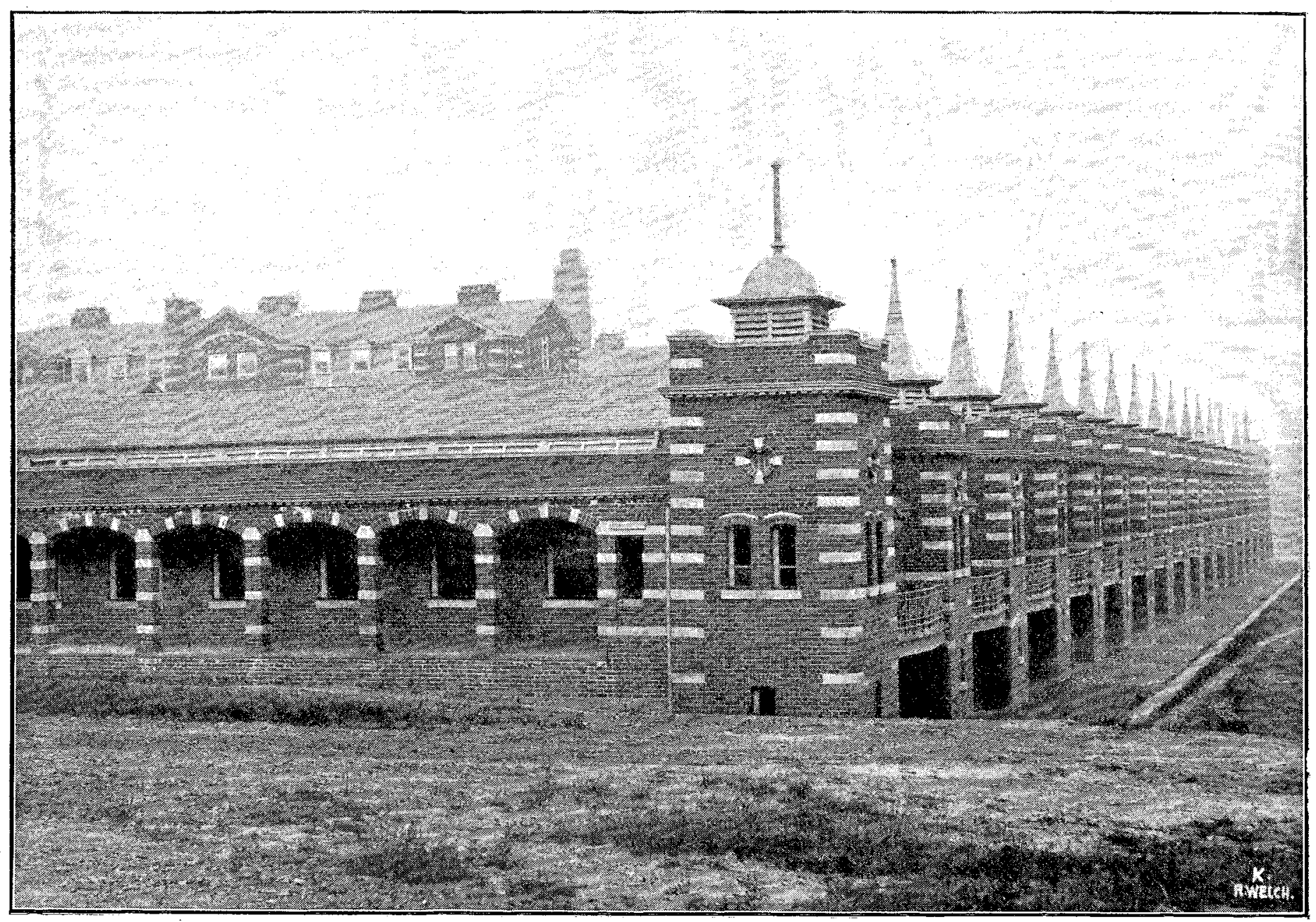

West arcade and south front, showing upper part of west administrative block and sanitary turrets at the south ends of the wards.

In reaching the entrance to the ward the branch air-duct divides into two and feeds the air entrances in each wall. The exit channels from the wards and corridors end in sanitary turrets at the south ends of the wards where the air escapes through flapping louvre windows. At the entrance of the branch air-ducts from the main air-duct more steam. heated pipes are placed. The quantity of steam entering these secondary pipes can be regulated so that for instance, one ward may be kept at $70^{\circ} \mathrm{F}$. and the adjoining one at $60^{\circ}$. In hot summer weather no steam will pass through the pipes. A change of air seven times an hour in winter and ten times in summer has been allowed for the whole building.

3. Owing to the adoption of the one storey system in the wards the necessity for staircases and passenger-lifts is avoided and labour to the nursing and medical staff is immensely minimised. Even in the arrangements for providing the patients with warm food, so often defective in physicians being used for clinical teaching; and each physician and each surgeon are provided with a clinical laboratory. The gynæcological and ophthalmic wards, small and large, form distinct units, complete in themselves, with operating theatres and kitchens. There are thus in all six operating theatres, an arrangement which will facilitate the amount of work to be done, will encourage individuality and healthy rivalry, and will enable students to see the details of surgical technique in a most complete way. The furnishing of the wards and theatres is very thorough, all the recent advances to bring about the fullest asepsis being adopted. The beds are iron white enamelled and the greater number of them are so constructed that they can be raised or lowered at the head. The wards are all on the ground floor and, with the exception of the eye block, are on the south side of the main corridor, which is a noble structure 460 feet long by 10 feet broad. The wards are lighted from the top by vertical "lantern" lights and by a window at the end. 
On the other side of the main corridor, at right angles to it, are the two administrative blocks, each with four storeys and a basement. In the western of these blocks are the kitchens, domestic offices, and nurses' and matron's quarters, while in the eastern block are the dispensary, day-rooms for resident medical staff, electrical rooms, "dark" room for photography, and bedrooms.

The out-patient department comprises an immense central waiting hall, the extern hall, 90 feet by 38 feet, with terrazzo floor and tiled walls. On either side are consulting rooms for medical, surgical, gynæcological, and ophthalmic cases There are also connected with this great out-patient hall an emergency room and operating theatre, lavatories, and sitting rooms for the medical men and nurses. Below the extern hall are a large cloak-room and lavatory accommodation for students with a special staircase running to the central or main corridor.

There are a large pathological department, a mortuary with for the marvellous success of this great undertaking belongs of right to the Right Hon. W. J. Pirrie and Mrs. Pirrie who have devoted an enormous amount of time, thought, and work to it. It is to their untiring industry and unselfish devotion that in this new Royal Victoria Hospital we possess so perfect a specimen of constructive art as applied in a thoroughly practical way to the science of healing. Mr. Pirrie has brought to bear on every detail in the construction of this new hospital from its inception until its completion the experience and specialised technical knowledge which he has gained in his own particular branch of business, while Mrs. Pirrie has in the fullest way utilised her acquaintance with the administrative management of a hospitail in its domestic and nursing relations. In addition to the mmense sum of money collected by Mrs. Pirrie for the building $(£ 100,000)$ and endowing $(£ 50,000)$ of the hospital, and apart altogether from what she and her husband so generously contributed themselves (a very large sum when it is all

FIG. 3.

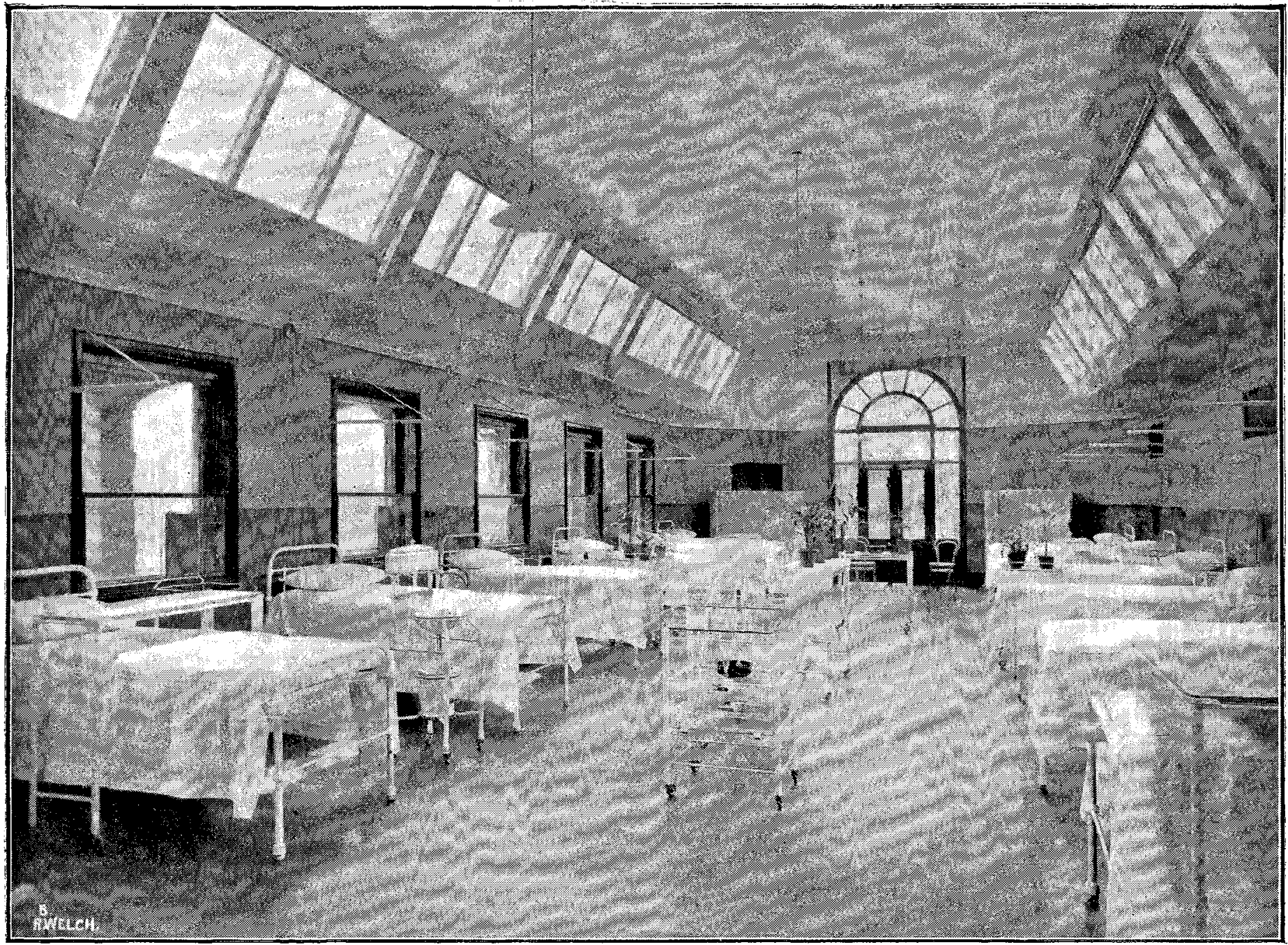

A medical ward, showing lantern or continuous roof windows, an end wintow of southera aspect, an 1 the openings on either side of the ward for the entrance of air.

a view-room for jurymen, a post-mortem theatre to hold 70 students, with a small laboratory and museum en suite, two isolation blocks, and on the east side a thoroughly equipped laundry with the most modern machinery. Electric light is used throughout the building and an electric room is being furnished with Roentgen ray apparatus and arrangements for cautery and light treatment, while the most modern filters and ingenious methods of using steam from the boilers for sterilising dressings, instruments, \&c., have been adopted.

There will be 12 resident medical officers, 17 members of the visiting staff (every specialty being represented) and an auxiliary staff (who hold office for two years). 76 nurses and 32 servants will be employed.

5. While the construction committee and the medical staff have done their utmost to make the new Royal Victoria Hospital worthy of the traditions of Belfast, the real credit computed), they have crowned all their other deeds of charity by spending a very large sum on the furnishing of the wards.

The medical and surgical staff recognise to the fullest degree that in every detail their arrangements have been met by Mr. and Mrs. Pirrie in a way hitherto absolutely unknown in public hospitals. They believe that there is not for its size $(300$ beds) a more perfect example of a modern hospital than this new one in Belfast, nor one in which the main considerations in the planning, construction, and furnishing of a hospital associated with a medical school in a large city are so fully and amply fulfilled.

Other names to be mentioned in connexion with the institution are those of Messrs. Henman and Cooper of Birmingham, the architects ; of Mr. Henry Lea, the consultng engineer; and of Messrs. McLaughlin and Harvey of Belfast, the builders. The cost of the building per bed will 
not amount to much more than $£ 300$. This estimate, how- $/$ hospital, as well as of the people of Belfast, His Majesty the ever, does not include the furnishing of the wards, while the site was free, and Mr. and Mrs. Pirrie paid the sum for buying out a public-house which was situated in one corner.

To the great satisfaction of all connected with the King is formally to open the new Victoria Hospital, erected to do honour to his distinguished and revered mother, on Monday next, July 27th. The gynæcological ward is to be named the Clarence Ward by Her Majesty the Queen in memory of the late Duke of Clarence.

FIG. 4.

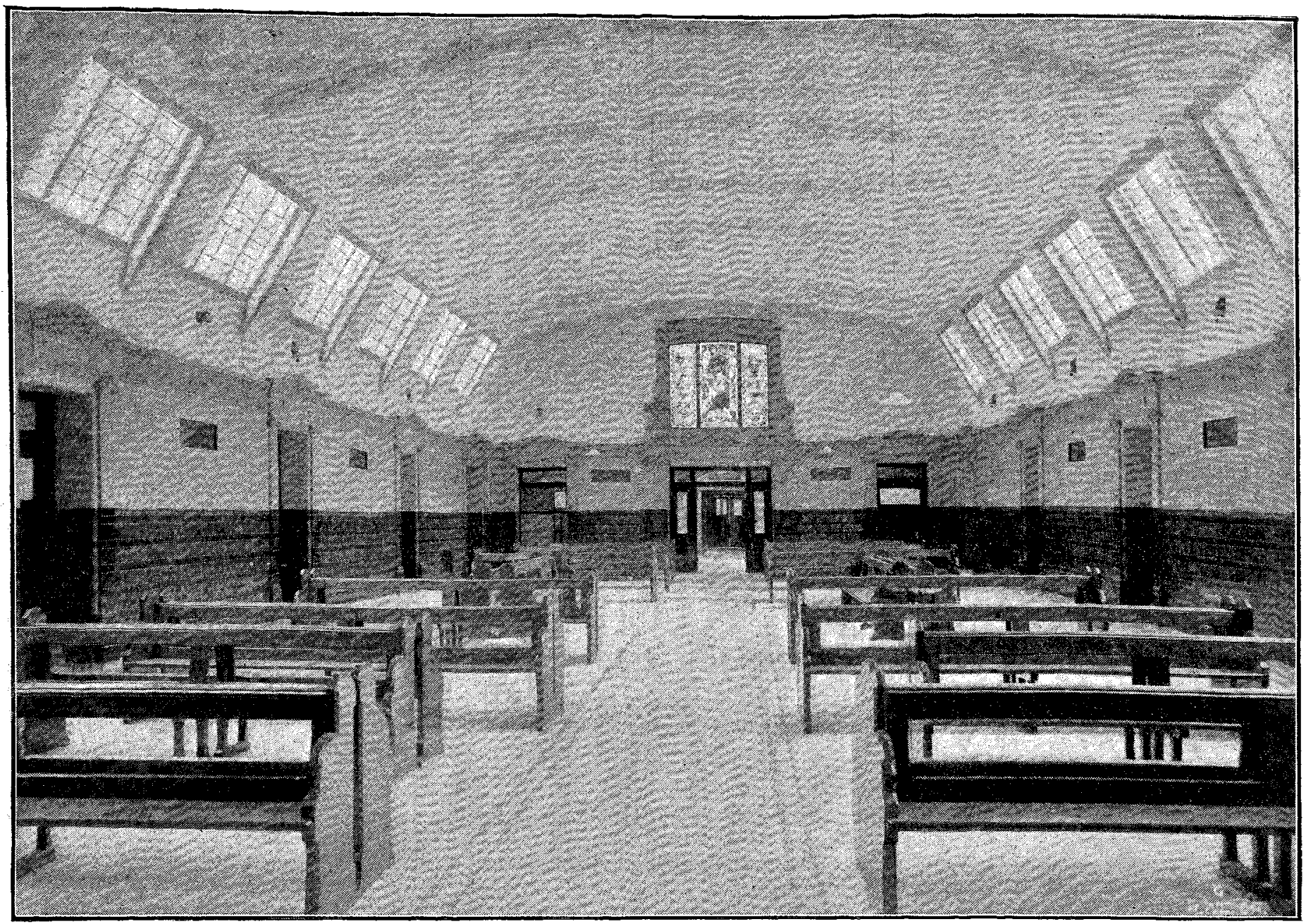

The Extern Hall.

\section{THE PREVENTION OF CONSUMPTION AND OTHER FORMS OF TUBERCULOSIS.}

Victoria Hospital for Consumption, EDINBURgh.

THE three new pavilions erected in the grounds of Craigleith House in connexion with the Victoria Hospital for Consumption were formally opened by Lord Rosebery on the afternoon of July 15th. The proceedings took place in a marquee erected in the grounds. The chair was occupied by the Lord Provost of Edinburgh, Sir James Steel, and there was a large attendance of well-known medical men and other prominent residents.

At present there are fally 70 patients in residence undergoing treatment. Craigleith House and a small annexe on its west side do not afford anything like sufficient sleeping accommodation for this large number of cases and, as a matter of fact, there are only 23 beds for indoor patients. The rest of the accommodation for sleeping purposes is found in a series of outdoor shelters arranged in circular form in the park in front of the house. This park, which is of considerable extent, is on a sunny slope facing south; it is completely encircled by a protecting belt of trees under which the patients can walk when the sun is strong. The patients of both sexes are quite reconciled to this outdoor life. The shelters, which are completely open on one side, each contain a couple of beds, and that there is no hardship in sleeping out under such conditions is evident from the fact that the resident physician at the hospital has elected to have a shelter for himself rather than a bed indoors.

The block plan of the extension shows a double row of " $\mathrm{Y}$ "-shaped pavilions-four in each row, with an administrative block behind. The state of the funds at present has only permitted of three of these being built. The pavilions are one storey in height, built of brick, with the floor about four feet above the ground. All the windows are of the steel casement type so that they can be opened to their fullest capacity. The four-bed ward is 29 feet by 16 feet by 12 feet in height and the six-bed ward is 29 feet by 23 feet by 12 feet in height. The floor, which is carried on iron beams, is of cement with wood on the top of it. There is a bath-room for every four patients, daily bathing being considered an important part of the treatment. The rooms are heated by open fires only, though there are hot-water pipes for the bath-rooms and sinks, and are fitted throughout with the electric light, as are also the shelters in the grounds. The smallest of the three pavilions-that with eight bedsnow built is the gift of Mr. W. Younger, M.P. (for the Stamford division of Lincolnshire), Auchen Castle, Moffat. The hospital is free to all up to its accommodation with the exception of eight beds which are reserved for those who can pay to the fund $£ 11$ s. per week. The extensions are from the designs of Messrs. Sydney Mitchell and Wilson, architects, Edinburgh.

The LORD PROvost said that he thought that the citizens of Edinburgh would be pleased when they saw the large amount of work which was being done in the new pavilions. Work of this kind could not be carried on without funds and he made an earnest appeal for contributions in aid of the 